\title{
A locally active discrete memristor model and its application
}

\section{in a hyperchaotic map}

\author{
Minglin Ma ${ }^{* 1}$, Yang Yang ${ }^{1}$, Zhicheng Qiu ${ }^{1}$, Yuexi Peng ${ }^{2}$, Yichuang Sun $^{3}$, \\ Zhijun Li ${ }^{1}$, Mengjiao Wang ${ }^{1}$ \\ 1. School of Automation and Electronic Information, Xiangtan University, Xiangtan, Hunan 411105, P.R.China \\ 2. School of Computer Science \& School of Cyberspace Science, Xiangtan University, Xiangtan, Hunan 411105 , \\ P.R.China \\ 3. School of Engineering and Technology, University of Hertfordshire, Hatfield AL10 9AB, UK
}

\begin{abstract}
The continuous memristor is a popular topic of research in recent years, however, there is rare discussion about the discrete memristor model, especially the locally active discrete memristor model. This paper proposes a locally active discrete memristor model for the first time and proves the three fingerprints characteristics of this model according to the definition of generalized memristor. A novel hyperchaotic map is constructed by coupling the discrete memristor with a two-dimensional generalized square map. The dynamical behaviors are analyzed with attractor phase diagram, bifurcation diagram, Lyapunov exponent spectrum, and dynamic behavior distribution diagram. Numerical simulation analysis shows that there is significant improvement in the hyperchaotic area, the quasi-periodic area and the chaotic complexity of the two-dimensional map when applying the locally active discrete memristor. In addition, antimonotonicity and transient chaos behaviors of system are reported. In particular, the coexisting attractors can be observed in this discrete memristive system, resulting from the different initial values of the memristor. Results of theoretical analysis are well verified with hardware experimental measurements. This paper lays a great foundation for future analysis and engineering application of the discrete memristor and relevant the study of other hyperchaotic maps.
\end{abstract}

Key words: Discrete memristor; Locally active; Hyperchaos; Coexisting attractors; Chaotic map

\section{Introduction}

The concept of the generalized memristor was firstly proposed by Chua in 1971[1]. Its appearance filled the gap between the electric charge and magnetic flux, and became the fourth basic circuit element. Then in 2008, Hewlett-Packard (HP) Laboratories successfully manufactured the real memristor using $\mathrm{TiO} 2$ and nano-level technology [2], which physically proved the existence of the memristor. On this foundation, the memristor attracts extensive attention from scientific communities. Due to the non-linear characteristics and memory function of memristor, it has been widely applied in the fields of nanotechnology [3, 4], neural network [5-8], circuit design [9-12], and secure communication [13], etc. It is worth noting that adding memristor in chaotic system can

\footnotetext{
* Corresponding author.

E-mail address: minglin_ma@xtu.edu.cn
} 
enhance chaos complexity and memristive coupling maps can generate hyperchaos [14], which provides a new research direction for the design of hyperchaotic systems.

The continuous memristor has been extensively studied for many years. Since locally active memristors have more complex dynamic characteristics than passive ones, they have a wider range of applications. In recent years, some locally active memristor models have been proposed and applied. For example, Wang et al. [15] proposed a locally active memristor and combined it with a capacitor and an inductance circuit to realize the simplest chaotic circuit. In the same year, Li et al. [16] realized a bistable local active memristor. Weiher et al. [17] further proved the existence of locally active memristors from the perspective of material physics experiments. Lin et al. [18] introduced the locally active memristor into the artificial neuron model and proposed a locally active memristive neuron model. A bistable locally active memristor was proposed in [19], and the coexisting firing patterns and phase synchronization in locally active memristor coupled neurons with HR and FN models were investigated. Although some locally active memristors have been reported, they still have not been used in discrete systems because of their complex mathematical models and small active intervals.

Therefore, it should be noticed that there is rare discussion about the discrete-time memristor model, especially the locally active discrete memristor model. On the one hand, the discrete memristor is more conducive to its application in discrete domain systems and digital circuits. Moreover, due to easy logic programming and higher accuracy, digital circuits have an important position in actual engineering. Therefore, the design of the discrete-time memristor mathematical model may become another important way to realize the memristor in the future. On the other hand, due to its unique iterative method, discrete memristors also provide a new direction for applications in secure communication and image encryption. Recently, Chew [20] proposed a discrete memristor based on $\mathrm{ZnO}$ nanowires synthesized on printed circuit board, but they did not provide the corresponding mathematical model. Karthikeyan [21] proposed the concept of a fractional discrete memristor, then the discrete memristor and the chaotic system based on the discrete memristor were obtained by using the discrete algorithm. Later, the discrete memristor was applied in chaotic map [22-24] and hyperchaotic map [25, 26]. Peng et al. [27] derived the discrete model of HP memristor, but they did not study the locally active discrete memristor. It should be noted that the above discrete memristors conform to the definition of memristors, but there is still a lack of various memristor models for theoretical analysis.

Moreover, there is still a lot of space for designing novel chaotic maps based on discrete memristors, which is helpful to explore more application backgrounds of discrete memristors. Because the locally active memristor is more conducive to the application of memristor in chaotic systems, we believe that it should be interesting to investigate a locally active discrete memristorbased discrete chaotic map and its dynamics. As far as we know, there have been no reports about this in the literature. Moreover, the two-dimensional generalized square map (2D-GSM) is a classical 2D discrete-time chaotic system with simple structure. It is worth studying whether the performance of the 2D-GSM can be improved further by using the memory and nonlinear characteristics of the locally active discrete memristor. As a result, our main motivation is to study the dynamic behaviors of a locally active memristor coupled to a two-dimensional map. In fact, the dynamic behaviors of discrete-time chaotic system are universal and important in the fields ranging from mathematics to information security. 
The rest of this paper is organized as follows. In Section 2, the locally active discrete memristor model is presented, and its related characteristics are investigated in detail. Section 3 shows the dynamics analysis of the locally active discrete memristor-based 2D-GSM. Hardware circuit based on FPGA is developed and circuit experiments are provided in Section 4. Finally, we conclude the results and prospects for future research.

\section{Description and analysis of the locally active discrete memristor model}

The locally active discrete memristor model is obtained as

$$
\left\{\begin{array}{l}
i(n)=W[x(n)] u(n)=x(n) u(n) \\
x(n+1)=F(x, u)=\alpha f[x(n)]+\beta u(n) \\
f[x(n)]=\operatorname{sgn}[x(n)+1]+\operatorname{sgn}[x(n)-1]+\gamma x(n)
\end{array}\right.
$$

where $i(n)$ is the current of the memristor, $u(n)$ is the input voltage of the memristor, $W[x(n)]$ is the memductance value of the memristor, $F(x, u)$ represents the internal state equation of the memristor and $\alpha, \beta, \gamma$ are three memristive parameters. In this paper, we set $\alpha=0.1, \beta=1$ and $\gamma=9$.

In order to verify the three fingerprints characteristics of the locally active discrete memristor model, let $u(n)$ in Eq. (1) be equal to $A \sin (2 \pi \omega n)$, that is, a sinusoidal discrete voltage signal is added to the discrete memristor model. And the volt-ampere curves of the locally active discrete memristor model are shown in Fig. 1. It shows that the volt-ampere curves present the " 8 "-shaped pinched hysteresis loops across the origin. As the amplitude $A$ increases from 3 to 5 , the area of the pinched hysteresis loop increases monotonously. As the frequency $\omega$ increases from 0.0001 to 0.0005 , the area of the pinched hysteresis loop decreases monotonously. In addition, when the frequency increases to infinity, the pinched hysteresis loop will shrink to a single-valued function across the origin. After the above analysis, the proposed locally active discrete memristor model conforms the definition of generalized memristor [28].

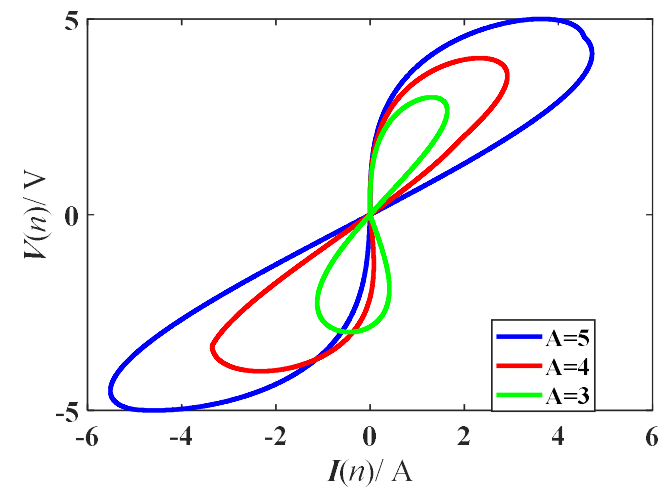

(a)

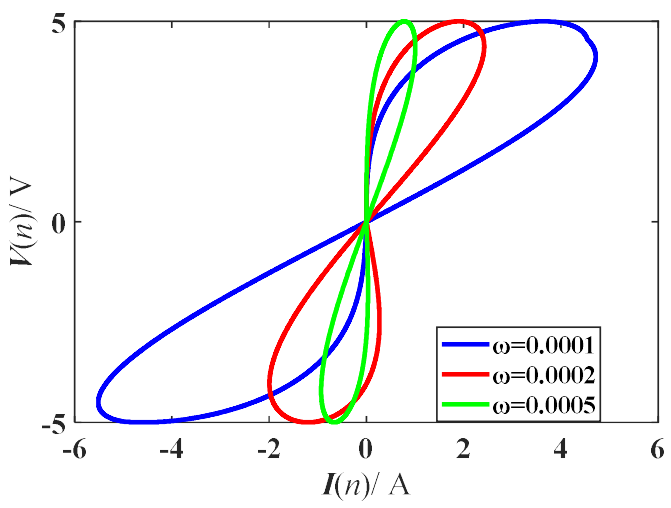

(b)

Fig. 1 Pinched hysteresis loops of the locally active discrete memristor model in Eq. (1). a The corresponding parameters are fixed at $\omega=0.0001$ and $A=5,4,3 \mathbf{b}$ The corresponding parameters are fixed at $A=5$ and $\omega=$ $0.0001,0.0002,0.0005$

Moreover, non-volatility is also an important characteristic of memristors. Power-off plot (POP) is usually used to characterize whether the circuit device is non-volatile. In order to verify the nonvolatility of the locally active discrete memristor, let $u(n)=0$, its internal state equation is simplified to 


$$
x(n+1)-x(n)=\alpha\{\operatorname{sgn}[x(n)+1]+\operatorname{sgn}[x(n)-1]-x(n)\}
$$

The POP curve in the $x(n+1)-x(n)$ versus $x(n)$ plane of the locally active discrete memristor is shown in Fig. 2a. In Fig. 2a, there are three negative slope intersections with the $x$-axis in the POP curve of the locally active discrete memristor. Consequently, according to the non-volatile memristor theorem, the proposed locally active discrete memristor model is a non-volatile memristor. On the other hand, there are a total of five points intersecting the $x$-axis in the POP curve of the discrete memristor, indicating that the memristor has five equilibrium points. According to the judgment method of Ref. [29], the equilibrium points $Q 1, Q 3$ and $Q 5$ with negative slopes are asymptotically stable, while the equilibrium points $Q 2$ and $Q 4$ with positive slopes are unstable. Thus, when the discrete memristor is given different initial states $x(0)$, the memristor gradually approaches three stable equilibrium states as shown in Fig. 2b, that is to say

$$
\begin{cases}X=x(Q 1)=-2, & \text { if } \quad x(0)<-1 \\ X=x(Q 3)=0, & \text { if } \quad-1<x(0)<1 \\ X=x(Q 5)=2, & \text { if } \quad x(0)>1\end{cases}
$$

Obviously, Fig. 2b and Eq. (3) indicate that the state $x(n)$ of the discrete memristor is different from the initial state $x(0)$, which shows its non-volatile properties.

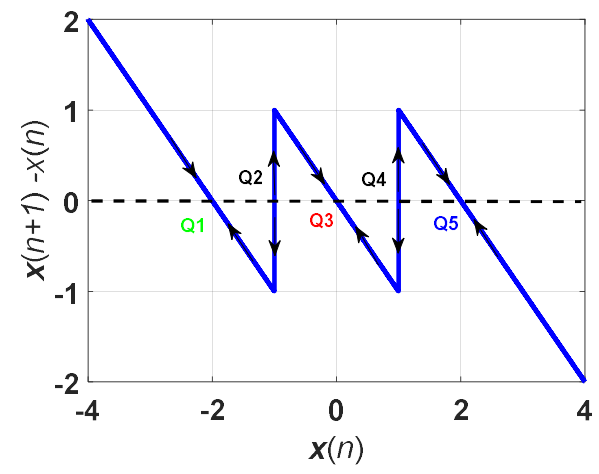

(a)

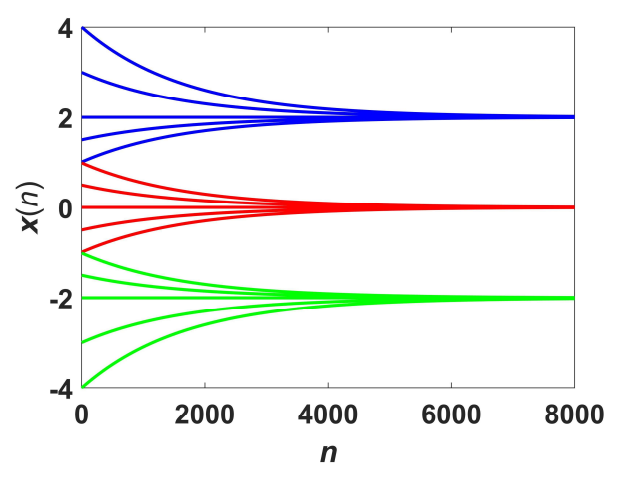

(b)

Fig. 2 Nonvolatile memory of the locally active discrete memristor: a POP with five equilibria and $\mathbf{b}$ an asymptotically tri-stable memory plot

In addition, it is also necessary to verify the property of local activity by observing the memristor DC $V-I$ plot. If there is a negative slope region in the DC $V-I$ plot, the memristor is called a locally active memristor [29]. In order to measure the DC $V-I$ trajectory of the locally active discrete memristor, set $x(n+1)-x(n)=0, x(n)=X$ and $u(n)=V$, then Eq. (1) derives its balance equation, as follows

$$
V=0.1 \times[X-\operatorname{sgn}(X+1)-\operatorname{sgn}(X-1)]
$$

where $V$ denotes $D C$ voltage, and $X$ is a variable equilibrium state satisfying $x(n+1)-x(n)=0, x(n)$ $=X$. Then, substituting Eq. (4) into Eq. (1), the $D C$ current $I$ can be calculated as

$$
I=X V=0.1 \times X[X-\operatorname{sgn}(X+1)-\operatorname{sgn}(X-1)]
$$

Considering Eqs. (4)-(5), the $D C V-I$ plot of the tri-stable locally active discrete memristor is drawn with the input DC voltage $V$ value varying from -1 to $1 V$ and the variable $X$ value varying within $(-2.2,2.2)$, as shown in Fig. 3a. It can be seen from Fig. 3a that the discrete memristor has three negative slope regions, so it is a locally active memristor. Moreover, the pinched hysteresis loops of the discrete memristor model with different initial values are shown in Fig. 3b. Therefore, 
it is worth studying whether the initial state of the locally active memristor can cause the memristive coupling system to produce coexistent attractors.

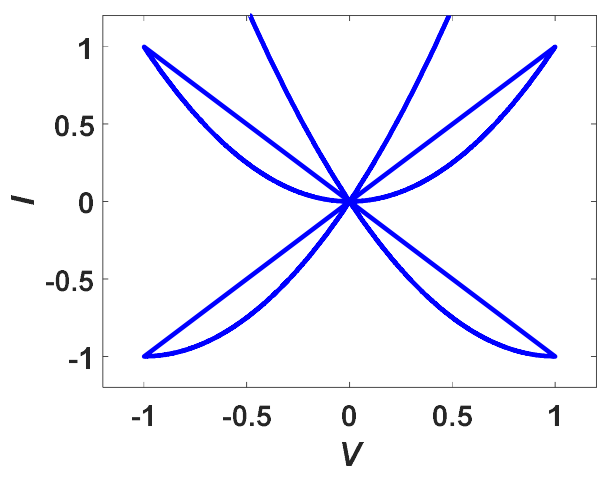

(a)

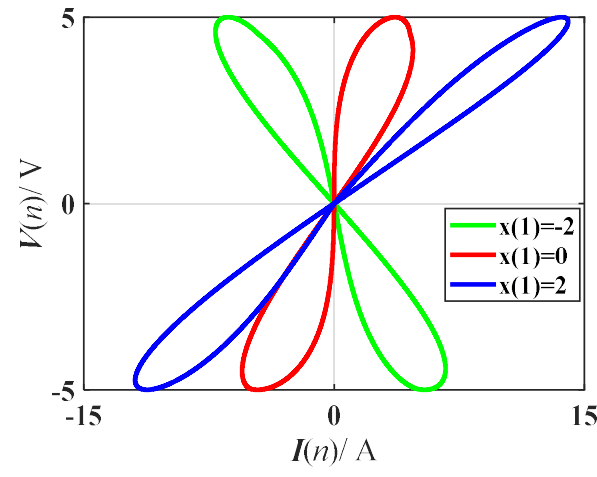

(b)

Fig. 3 The local activeness of the locally active discrete memristor: a DC $V-I$ loci associated with the equilibrium state on interval $-2.2<X<2.2 \mathbf{b} A=5, \omega=0.0001$ and different initial state-dependent pinched hysteresis loops

\section{Dynamics of the locally active discrete memristor-based hyperchaotic map}

3.1 The locally active discrete memristor-based hyperchaotic map

Generally, the two-dimensional generalized square map (2D-GSM) is obtained by extending the one-dimensional map. Its mathematical expression is

$$
\left\{\begin{array}{l}
x(n+1)=b\left\{\exp \left[-a x^{2}(n)\right]-x^{2}(n)\right\}+r y(n) \\
y(n+1)=b\left\{\exp \left[-a y^{2}(n)\right]-y^{2}(n)\right\}+r x(n)
\end{array}\right.
$$

where $x(n)$ and $y(n)$ denote variables of the system, $a, b$ and $r$ are system parameters.

When the locally active discrete memristor is coupled to the 2D-GSM, the locally active discrete memristive hyperchaotic map (DMHM) can be established and written as

$$
\left\{\begin{array}{l}
x(n+1)=b\left\{\exp \left[-a x^{2}(n)\right]-x^{2}(n)\right\}+r k y(n) z(n) \\
y(n+1)=b\left\{\exp \left[-a y^{2}(n)\right]-y^{2}(n)\right\}+r x(n) \\
z(n+1)=z(n)+\alpha\{\operatorname{sgn}[z(n)+1]+\operatorname{sgn}[z(n)-1]-z(n)\}+y(n)
\end{array}\right.
$$

where $a, b, r$ and $\alpha$ are system parameters and $k$ represents the coupling coefficient. In this paper, we set $a=10, \alpha=0.1$ and $k=0.1$.

The structure diagram of DMHM is shown in Fig. 4. The locally active discrete memristor is added to the original map for status feedback control. 


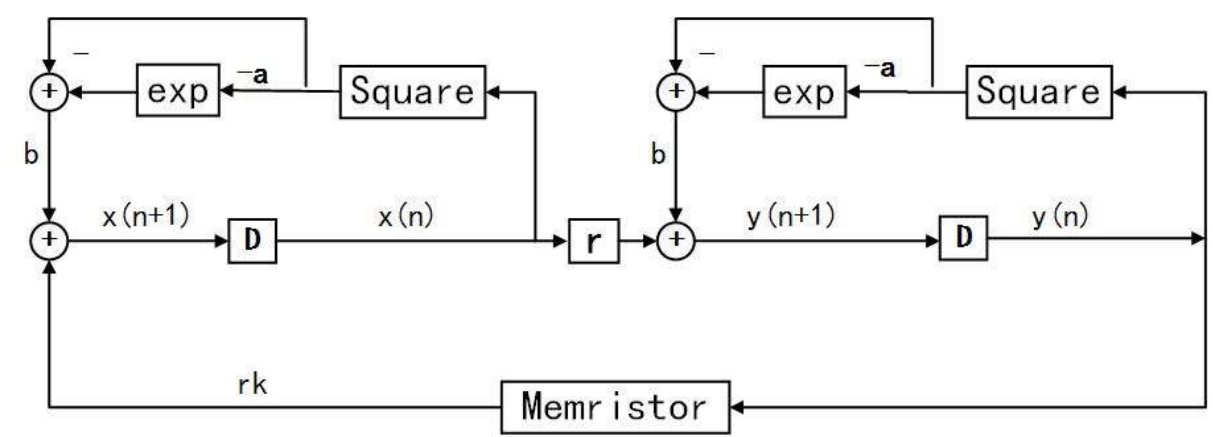

Fig. 4 Structure diagram of the DMHM

\subsection{Hyperchaotic region}

The chaotic attractors phase trajectories of the 2D-GSM and the DMHM with different parameters are shown in Fig. 5. When initial states are set as $x(0)=0, y(0)=0.1, z(0)=2$ and the parameter $b$ is set to 0.7 , the attractors phase trajectories of the 2D-GSM are separately chaotic, periodic, hyperchaotic, quasi-periodic state with the parameter $r=-0.8,-0.55,-0.1$ and 0.23 . However, the attractors phase trajectories of the DMHM are all hyperchaotic state with the parameter $r=-0.8$, $-0.55,-0.1$ and 0.23 . It can be seen that the DMHM expands the original hyperchaotic region of the 2D-GSM and becomes more complicated after adding the locally active discrete memristor.
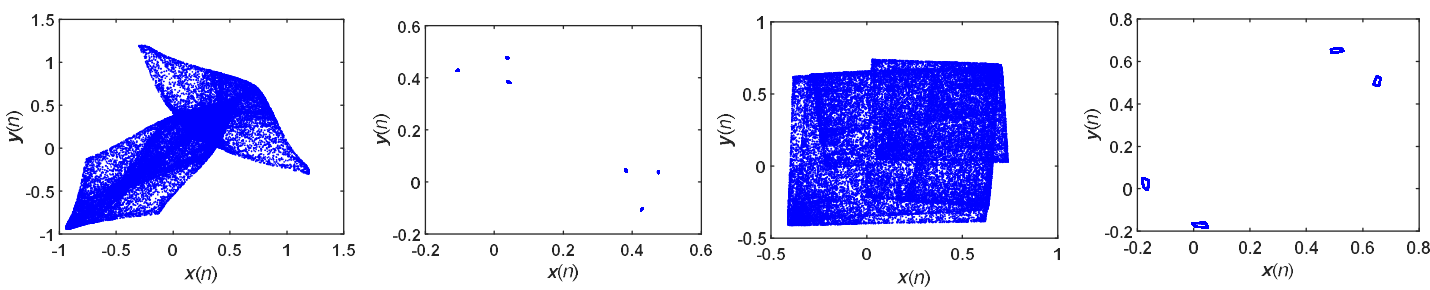

(a)
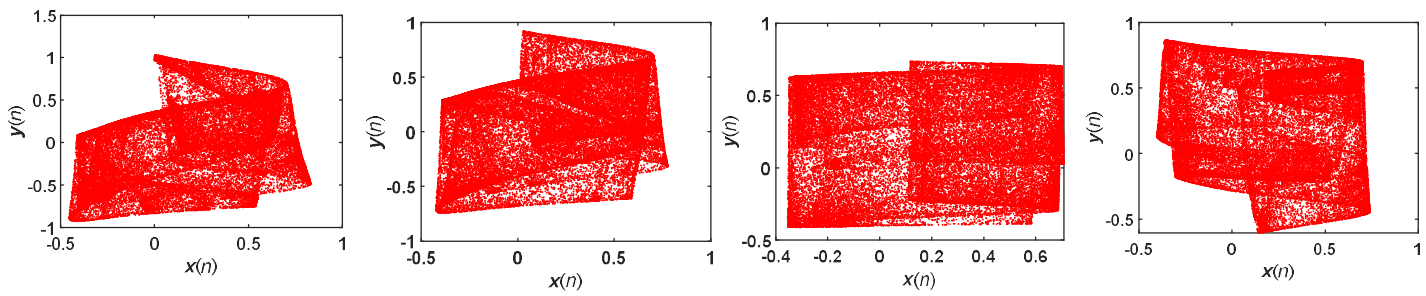

(b)
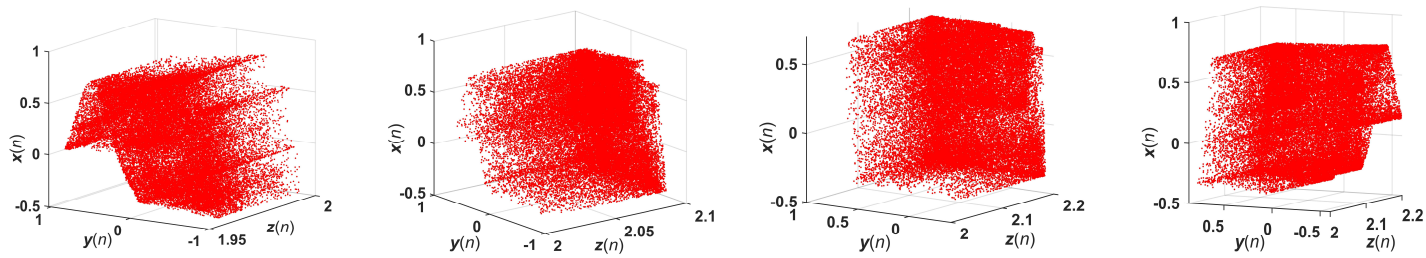

(c)

Fig. 5 Chaotic attractors phase trajectories with parameters $a=10, b=0.7$ and $r=-0.8,-0.55,-0.1$ and 0.23 of a the GSM with $x(0)=0, y(0)=0.1 \mathbf{b}$ two-dimensional attractor diagrams of the DMHM with $x(0)=0, y(0)=$ $0.1, z(0)=2 \mathbf{c}$ three-dimensional attractor diagrams of the DMHM with $x(0)=0, y(0)=0.1, z(0)=2$

Then, when initial states are set as $x(0)=0, y(0)=0.1, z(0)=2$, the parameters of the DMHM are still set as $a=10, k=0.1, b=0.7$ and the parameter $r$ is variable, the bifurcation and its 
corresponding LEs for the 2D-GSM and the DMHM are illustrated in Fig. 6, respectively. From Fig. 6 , it can be seen that the hyperchaotic region of the DMHM is wider than that of the original 2DGSM, and its hyperchaotic value range of parameter $r=(-1,0.537],(0.565,0.81]$ is obviously much wider than that of the 2D-GSM with $r=(-1,-0.69],(-0.56,-0.47],(-0.19,0.22]$. In other words, after adding the locally active discrete memristor, they are all in a hyperchaotic state, except for the only two extremely narrow period windows. These can also prove that the two-dimensional hyperchaotic map has expanded the original hyperchaotic region by adding the locally active discrete memristor. Therefore, the dynamic behaviors in the DMHM based on the locally active discrete memristor model are richer than that in the original one.

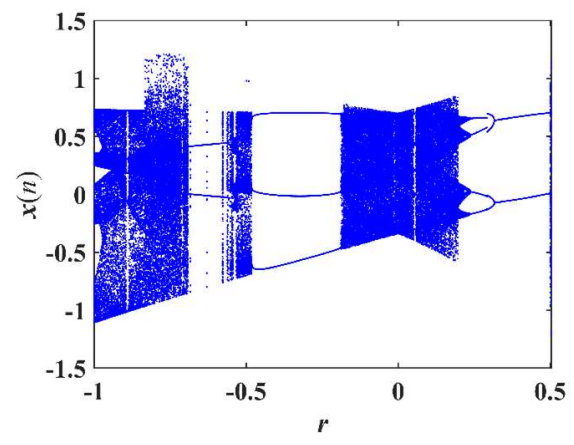

(a)

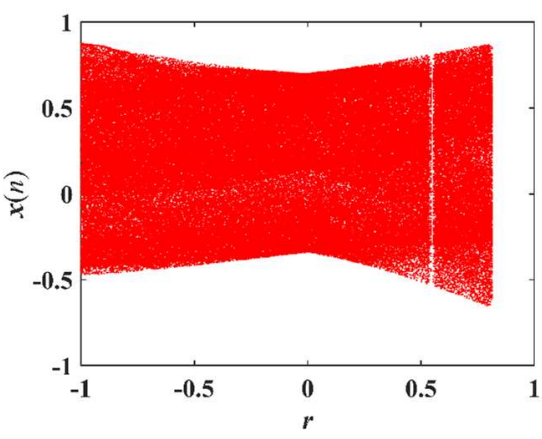

(c)

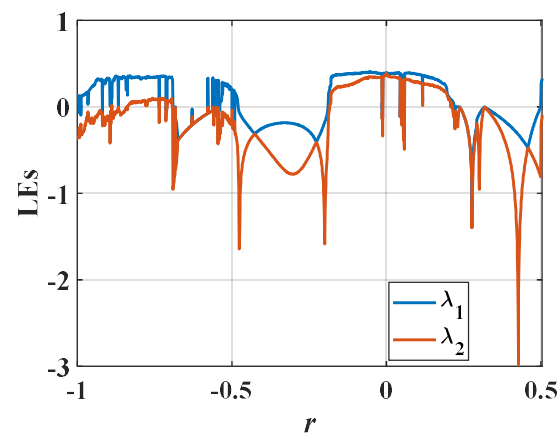

(b)

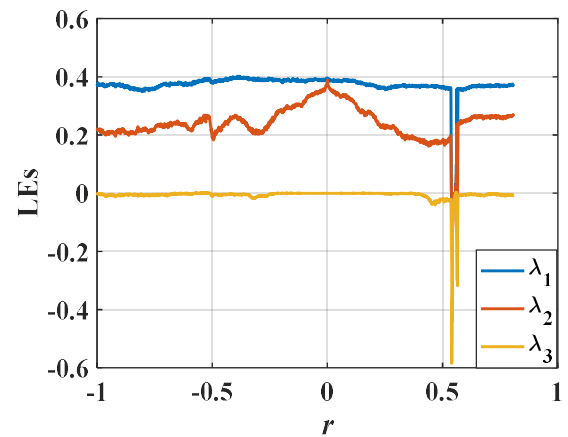

(d)

Fig. 6 Bifurcation and its corresponding LEs with $b=0.7$ and $x(0)=0, y(0)=0.1, \mathrm{z}(0)=2$ a bifurcation of the GSM with different $r=[-1,0.5]$ b LEs of the GSM with different $r=[-1,0.5]$ (c) bifurcation of the DMHM with different $r=[-1,1]$ (d) LEs of the DMHM with different $r=[-1,1]$

Furthermore, in order to measure the closeness of the chaotic sequence to the random sequence, the spectral entropy (SE) complexity measure algorithm [30] is used to calculate the complexity of the 2D-GSM and the DMHM. The darker color area in the SE complexity figure means the greater complexity and the more random of the chaotic sequence. Here, the sample size of the time series is set to 40,000. Afterward, the SE complexity of the 2D-GSM and the DMHM are plotted in Fig. 7, respectively. Comparing Fig. 7a and Fig. 7b, it can be seen that most areas of the original 2DGSM are low complexity areas. On the contrary, the DMHM has two large high complexity bands, and its high complexity area is much larger than that in the 2D-GSM. The low complexity areas in the 2D-GSM have all become to high complexity areas by coupling the locally active discrete memristor, which also corresponds to the bifurcation diagram in Fig. 6. It demonstrates that the DMHM system is more complicated after adding the locally active discrete memristor, and the oscillating signal generated is more random. Thereby the DMHM based on the discrete memristor 
model has better prospects in engineering applications, such as pseudo-random number generators or secure communications.

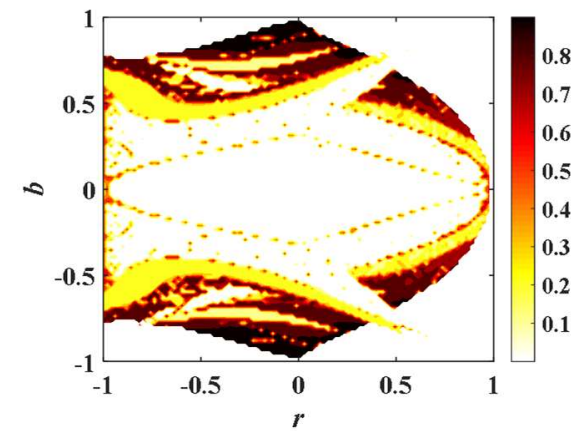

(a)

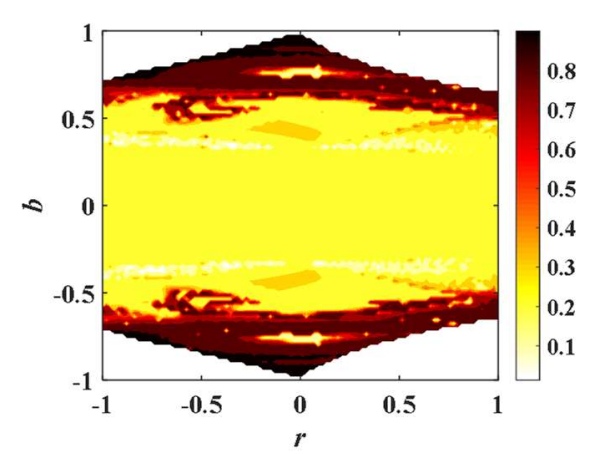

(b)

Fig. 7 SE complexity with $a=10$ and variable parameters $r=[-1,1]$ and $b=[-1,1]$ for a the GSM with $x(0)=$ $0, y(0)=0.1, \mathbf{b}$ the DMHM with $x(0)=0, y(0)=0.1, z(0)=2$

\subsection{Quasi periodic region}

When the parameter $b$ is fixed to $b=0.5$ and the parameter $r$ is variable, the bifurcation and its corresponding LEs for the 2D-GSM and the DMHM are illustrated in Fig. 8, respectively. As shown in Fig. 8, it can be seen that the quasi periodic region of the DMHM is wider than that of the original 2D-GSM. In original 2D-GSM, the quasi periodic value range of parameter $r$ is just $r=(0.256$, $0.273]$, but it is magnified about 16 times to $r=(0.326,0.61]$ in the DMHM. Although the bifurcation diagram corresponding to this fluctuation interval seems to be chaotic, the system is in a quasi periodic state. For example, when $r=0.6$, the attractor phase diagrams of the hyperchaotic system are given in Fig. 9a. It is shown that the attractor of this system is a circular ring, which proves the quasi periodic state of the DMHM. In addition, the DMHM exhibits quasi periodic and periodic states alternately before entering chaos, as shown in Fig. 9. It can be seen that the DMHM has expanded the original quasi periodic region by adding the locally active discrete memristor. Hence, the dynamic behaviors in the DMHM based on the discrete memristor model are richer than that in the original one. 


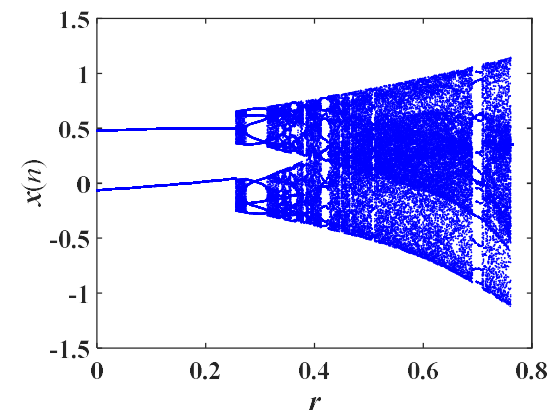

(a)

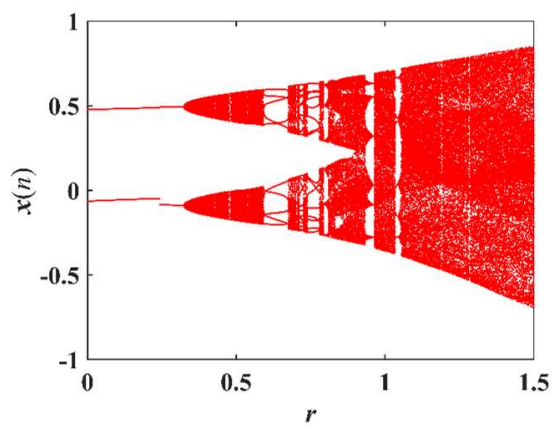

(c)

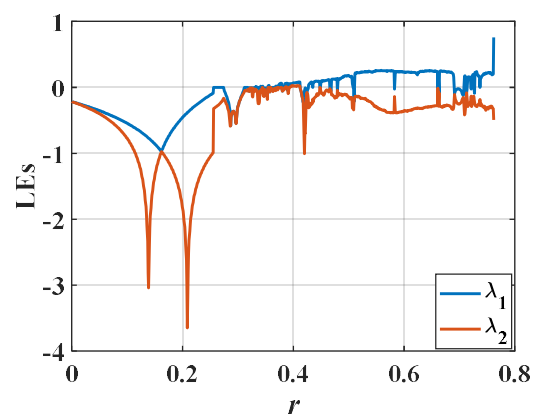

(b)

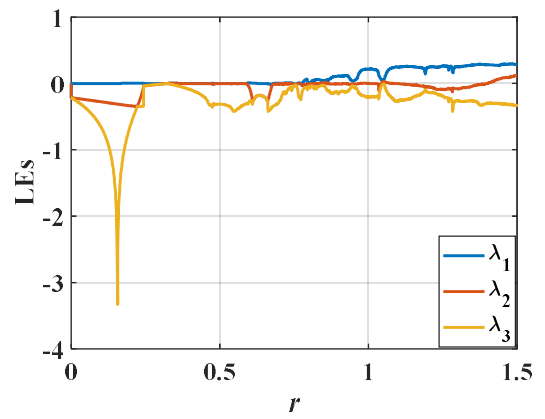

(d)

Fig. 8 Bifurcation and its corresponding LEs with $b=0.5$ and $x(0)=0, y(0)=0.1, z(0)=2$ a bifurcation of the GSM with different $r=[0,0.8] \mathbf{b}$ LEs of the GSM with different $r=[0,0.8] \mathbf{c}$ bifurcation of the DMHM with different $r=[0,1.5] \mathbf{d}$ LEs of the DMHM with different $r=[0,1.5]$

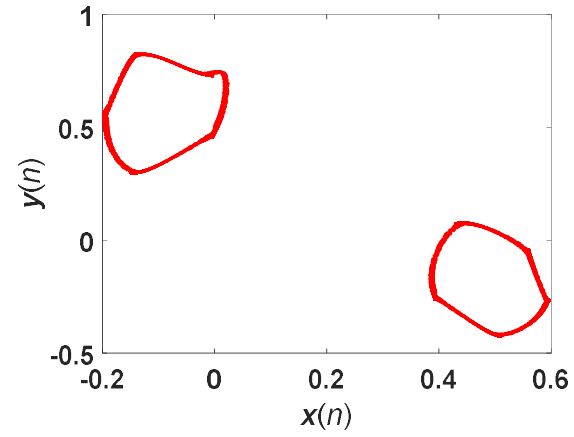

(a)

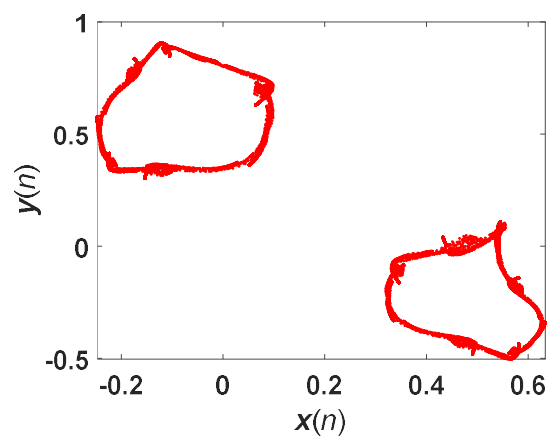

(c)

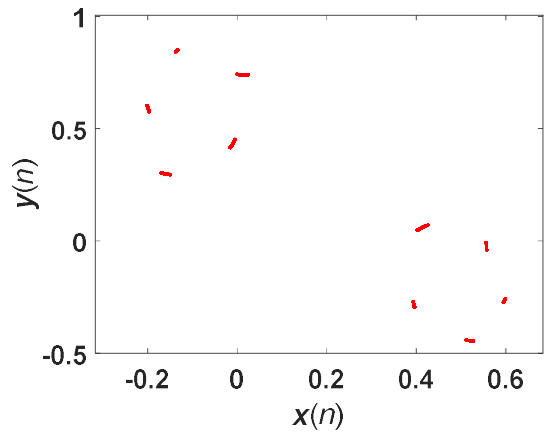

(b)

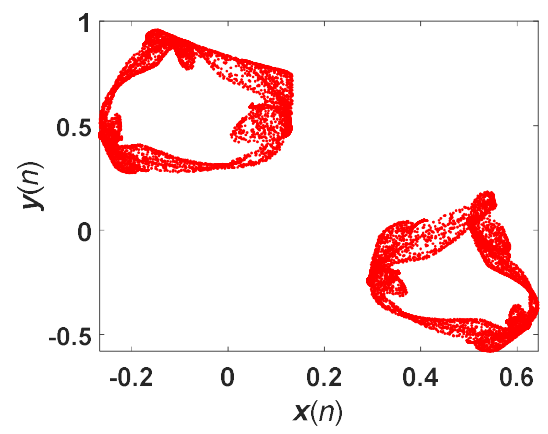

(d)

Fig. 9 Chaotic attractors phase trajectories of the DMHM with parameters $b=0.5$ and $\mathbf{a} r=0.6, \mathbf{b} r=0.65, \mathbf{c} r$ $=0.75, \mathbf{d} r=0.8$ 


\subsection{Coexisting attractors}

When the parameters are fixed at $a=10, b=0.5$ and parameter $r$ is variable, the bifurcation diagrams of the 2D-GSM and the DMHM are illustrated in Fig. 10a, b, respectively. In Fig. 10b, the bifurcation diagram of the DMHM with different initial values are represented in different colors, that is, blue, green, and red respectively represent the initial value $\left(\begin{array}{lll}0 & 0.1 & 2\end{array}\right),\left(\begin{array}{lll}0 & 0.1 & 0\end{array}\right)$ and $\left(\begin{array}{lll}0 & 0.1 & -2\end{array}\right)$. And when the parameters are fixed at $\mathrm{a}=10, \mathrm{~b}=-0.5, \mathrm{r}=1$ and the initial value $z(0)$ is variable, the bifurcation diagram of the DMHM is shown in Fig. 10c. It can be seen that the DMHM displays complex dynamical behaviors of period/ periodic windows, quasi-period, chaos and hyperchaotic behaviors, as can be observed from Fig. 10b. Moreover, the current states of the DMHM are three different states when $r$ takes a certain value, that is, the phenomenon of coexistence. With the value of $r$ ranging from -2.2 to 2 , the long-term behavior of the system depends crucially on the choice of initial conditions, thus leading to the interesting and striking phenomenon of coexisting multiple attractors' behavior. In addition, the effective value range of parameter $r=(-2.2,2]$ in DMHM is also much wider than that of the 2D-GSM $r=(-1,0.8]$, which means that the DMHM greatly expands the effective range of the 2D-GSM. This indicates that the introduction of locally active discrete memristor can greatly enhance the chaotic complexity and effective range of the original discrete map. And the state of the system can be adjusted by selecting different system initial values, which can be meaningfully applied in the field of secure communication.

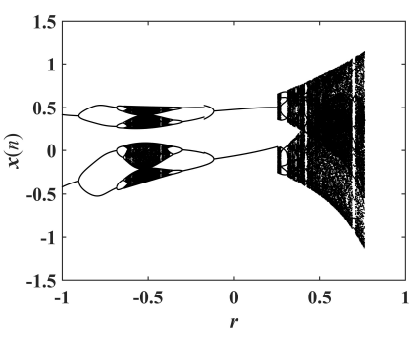

(a)

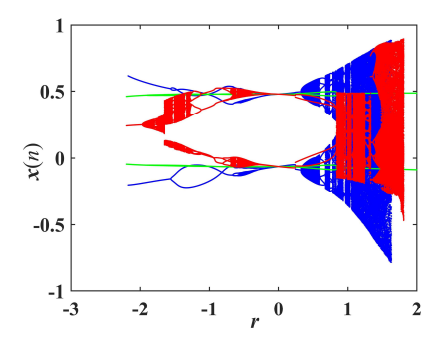

(b)

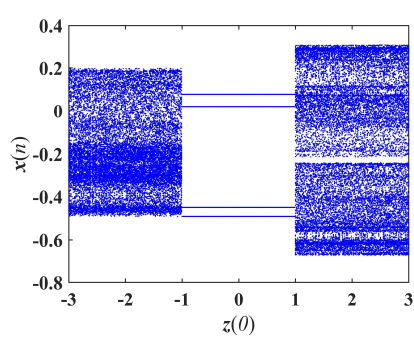

(c)

Fig. 10 a Bifurcation of the states $x(n)$ for the 2D-GSM with $a=10, b=0.5$, different $r=[-1,0.8]$ and $x(0)=$ $0, y(0)=0.1 \mathbf{b}$ bifurcation of the states $x(n)$ for the DMHM with $a=10, b=0.5$, different $r=[-2.2,2]$ and $x(0)$ $=0, y(0)=0.1, z(0)=2$ (blue), $x(0)=0, y(0)=0.1, z(0)=0$ (green), $x(0)=0, y(0)=0.1, z(0)=-2$ (red) $\mathbf{c}$ bifurcation of the states $x(n)$ for the DMHM with $a=10, b=-0.5, r=1$, different $z(0)=[-3,3]$

Correspondingly, without changing the parameters in Fig. 10b, the attractor phase diagrams with different initial values are plotted in Fig. 11, where blue, green, and red relate to the initial conditions selected from the corresponding regions of Fig. 10, respectively. Fig. 11a shows that the system is chaotic when the initial value represented by red is selected, while it shows several fixed points with initial values represented by green and blue. Fig. $11 \mathrm{~b}$ shows that the system is quasi-periodic when the initial value represented by blue is selected, while it shows several fixed points with initial values represented by green and red. Fig. 11c shows that the system is chaotic when initial values represented by blue and red are selected, while it shows several discrete line segments with the initial value represented by green. Therefore, the phase diagram shows a variety of complex coexistence phenomena, which is consistent with the situation illustrated in Fig. 10. Thus, the phenomenon of coexisting attractors is confirmed in the discrete memristive hyperchaotic system. 


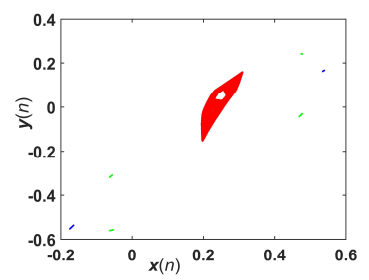

(a)

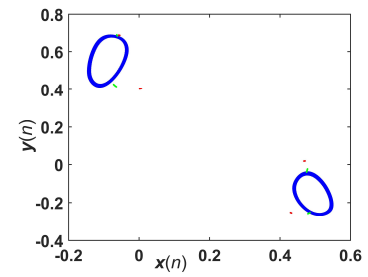

(b)

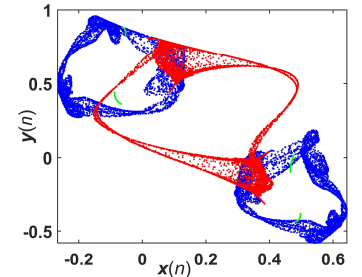

(c)

Fig. 11 Coexisting attractors of the DMHM with different initial values and parameters a $a=10, b=0.5, r=$ $-1.7, \mathbf{b} a=10, b=0.5, r=0.4, \mathbf{c} a=10, b=0.5, r=0.8(x(0)=0, y(0)=0.1, z(0)=2$ (blue), $x(0)=0, y(0)=$ $0.1, z(0)=0$ (green), $x(0)=0, y(0)=0.1, z(0)=-2($ red $))$

Finally, when the system parameters are set at $a=10, k=0.9, r=1.3$ and the initial values $y(0)$, $z(0)$ are set as variables, the SE complexity of the DMHM is depicted in Fig. 12. The red or darker color indicates that the system generates chaotic or hyperchaotic behavior, while the yellow and white indicate that the system exhibits quasi-periodic or fixed points state. The existence of multiple colors in this figure proves that the state of the system is different when the initial value of the system is different. It also proves that the memristive hyperchaotic map has the phenomenon of coexisting attractors. Besides, these results imply that the bifurcation routes are closely related to the initial values, leading to the occurrence of the coexisting phenomenon.

As a conclusion, the system sensitivity is not only related to system parameters, but also greatly dependent on initial state values of the discrete memristor. Such initial value induced multi-stability has not previously been reported in discrete locally active memristor-hyperchaotic systems.

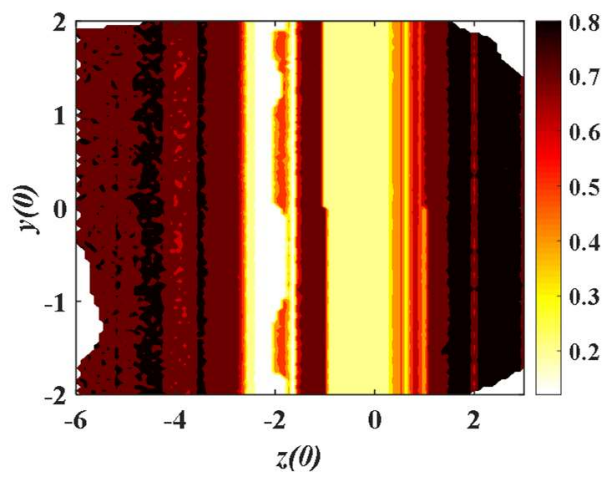

(a)

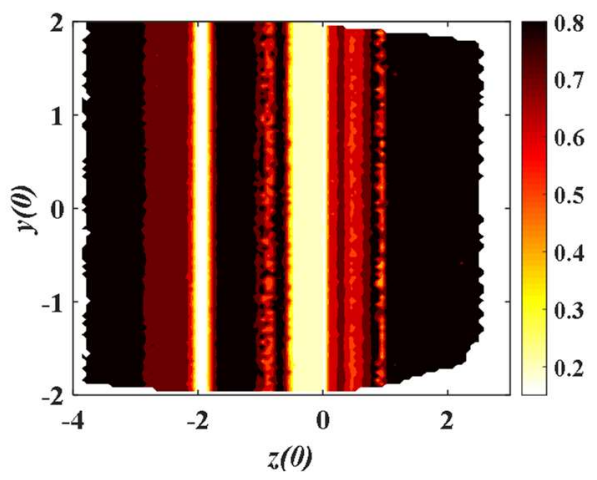

(b)

Fig. 12 SE complexity of the DMHM with variable initial values $y(0)$ and $z(0)$ and parameters a $r=1.3, b=0.5$, b $r=1.3, b=-0.55$

\subsection{Transient chaos behavior of system}

Under different iteration times, the time sequences of the system state $x(n)$ and its corresponding $x-$ $y$ phase plane plots are shown in Fig. 13, respectively. Noted that in Fig. 13a, b, the time series of system state $x(n)$ presents the phenomenon of transient chaos and transient period alternating within the time interval $n=(1,40000)$ colored in royal blue, and the whole is presented a hyperchaotic phase plane plot in the $x-y$ plane. Then, it is worth noting that in Fig. $13 \mathrm{c}, \mathrm{d}$, the time series of system state $x(n)$ exhibits periodic iteration within the time interval $n=(31000,32500)$ colored in royal blue, and the whole is shown as the period plane plot in the $x-y$ plane. Therefore, the DMHM 
has the phenomenon of transient chaos and transient period alternating in the time series, that is, the system presents different states due to different iteration times.

As is clear from the above analysis, the locally active memristive hyperchaotic system generates multiple patterns and multiple stabilities with the existence of the transient chaos and the transient period types of patterns. And the state form of multiple stabilities of the locally active memristive hyperchaotic system can be changed by choosing different iteration times, which greatly improves the complexity of the original two-dimensional map and is more conducive to use in secure communication.

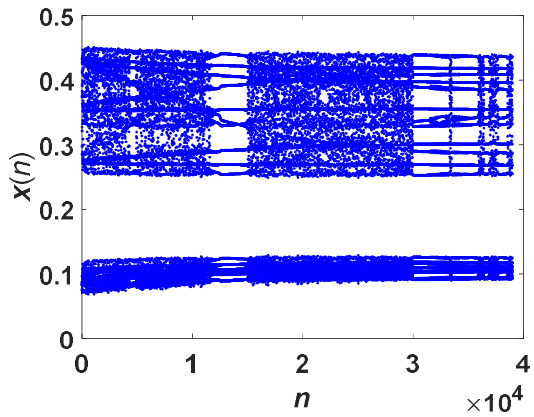

(a)

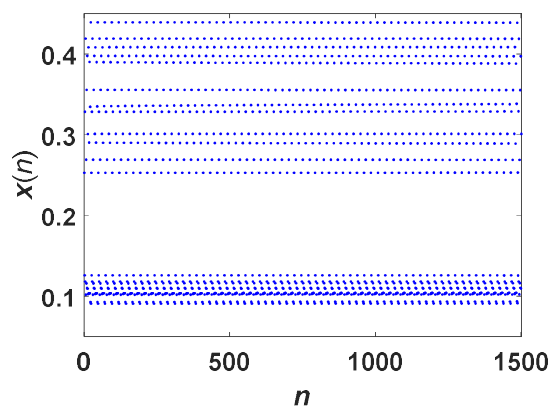

(c)

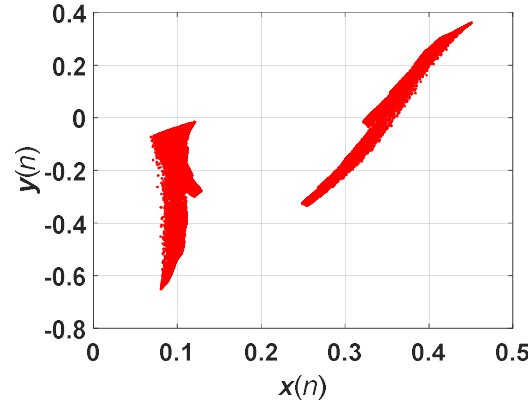

(b)

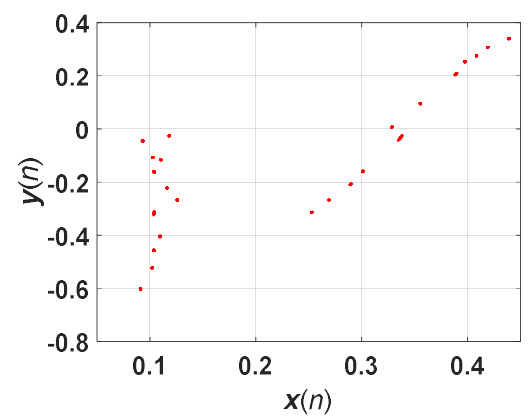

(d)

Fig. 13 The DMHM with parameters $b=0.5$ and $r=0.6$, a $x(n)$ sequence with parameter $n=[1,40000], \mathbf{b}$ attractor with parameter $n=[1,40000], \mathbf{c} x(n)$ sequence with parameter $n=[31000,32500]$, d attractor with parameter $n=[31000,32500]$

\subsection{Antimonotonicity}

Antimonotonicity refers to the phenomenon that periodic orbits can be generated and then disappear through reverse period doubling bifurcation with the monotonic change of a certain system parameter in various nonlinear systems [31]. Parlitz et al. [32-33] reported the phenomenon of antimonotonicity in the Duffing oscillator. And Kocarev et al. [34] showed that antimonotonicity is a typical phenomenon in Chua's circuit. Later, memristor-based jerk circuits [35] and 4-D chaotic system [36] also reported the phenomenon of antimonotonicity. The above systems are all continuous nonlinear systems. As a discrete system, the DMHM also produces the antimonotonic phenomenon with the monotonic increase of the system parameter $r$.

Also, in order to prove the phenomenon of antimonotonicity in DMHM, we have produced some bifurcation diagrams as system parameter $r$ is varied in the range of $-1.5 \leq r \leq 0$. And the ample results are depicted in Fig. 14. It can be seen that a period-8 bubble is observed at $b=0.45$ in Fig. 14a, and the branch develops a stable period- 16 bubble at $b=0.46$ in Fig. 14b. As $b$ is further 
increased to 0.47 , more bubbles are generated until an infinite tree (such as chaos) finally occurs in Fig. 14c. Similarly, we have another period- 8 bubble at $b=0.48$ in Fig. 14d. At the same time, there is also a bubble tree on the left side of the whole bubble tree at $b=0.5$ in Fig. 14e. In short, it can be seen that the DMHM has a complex bubble tree phenomenon, which is more complicated than the original system.

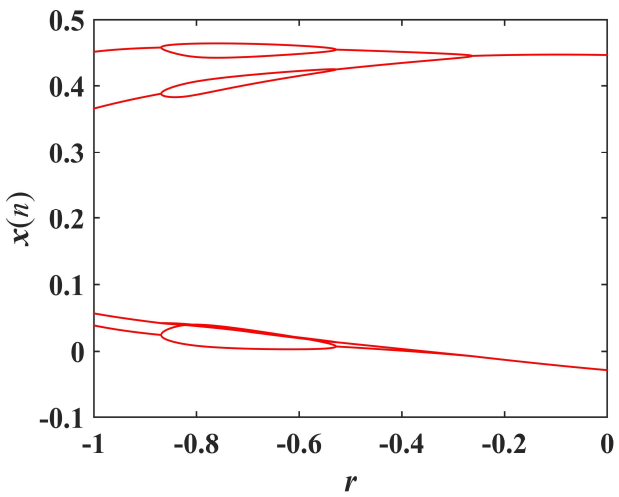

(a)

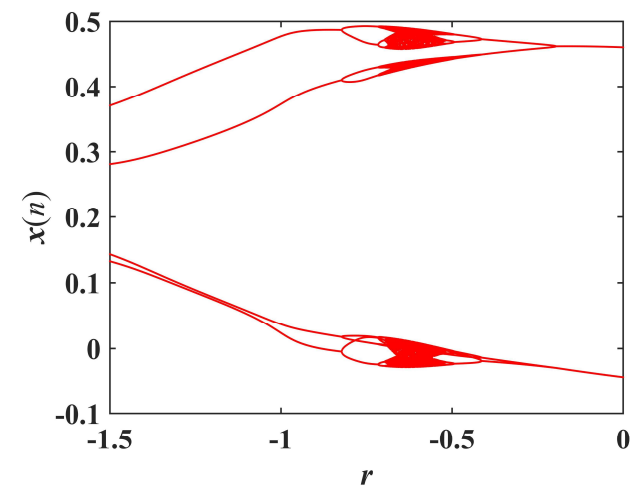

(c)

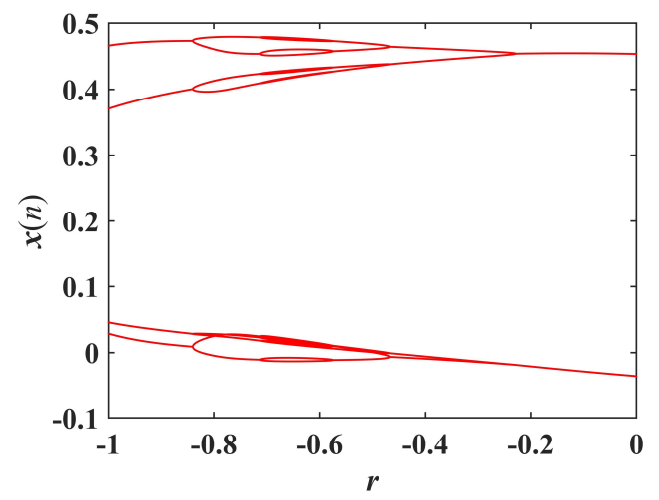

(b)

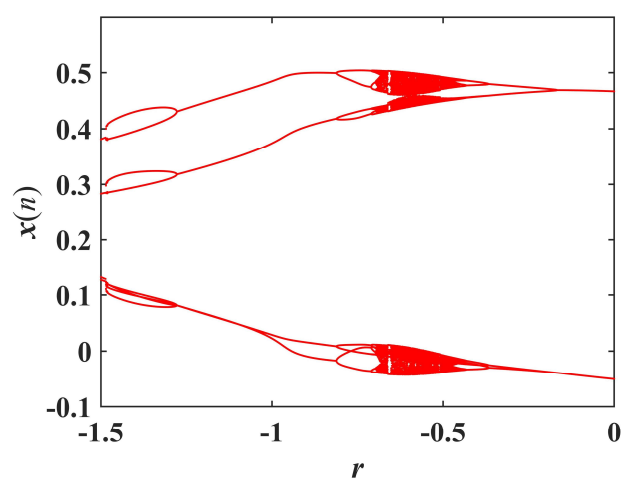

(d)

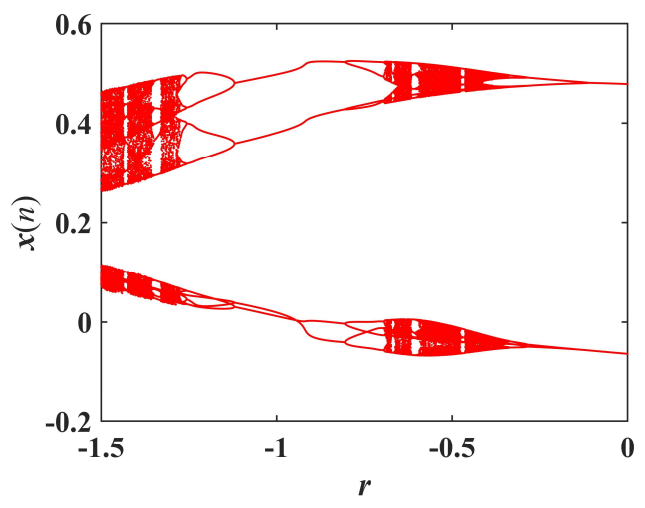

(e)

Fig. 14 Bifurcation with $x(0)=0, y(0)=0.1, z(0)=-2$ of the DMHM a with different $r=[-1,0]$ and $b=0.45$, b with different $r=[-1,0]$ and $b=0.46$, c with different $r=[-1.5,0]$ and $b=0.47$, d with different $r=[-1.5$, $0]$ and $b=0.48$, e with different $r=[-1.5,0]$ and $b=0.5$

Hence, it can be concluded that the DMHM has complex dynamics and its dynamical behaviors depend on the system parameters $r, b$ and initial values. The system sensitivity is not only related to system parameters, but also greatly dependent on initial state values of the discrete memristor. In 
summary, after the above analysis, the dynamic behavior in DMHM based on the locally active discrete memristor model is more abundant than that in the original model.

\section{Hardware implementation}

\subsection{Hardware circuit design}

Since the DMHM system is a 3D discrete dynamical system, it is more convenient and simpler to implement it in a digital hardware platform than in an analog hardware platform. So to validate the numerical results of the DMHM system, we design digital experiments using FPGA circuit boards on the hardware platform. The hardware platform consists of one Altera EP2C8Q208C8 as the core controller, one interface board with Video Graphics Array (VGA), and some peripherally-linked circuits, as can be observed from Fig. 15. In experimental measurements, the time series and phase trajectories of the DMHM system are captured on the display screen by the VGA output terminal.

\subsection{Experimental results}

In order to actually observe the chaotic signals generated from the DMHM, the digital experiment is practically implemented in Fig. 15. Following the results in Fig. 13, the transient chaos behavior of the DMHM is experimentally captured in Fig. 15. And, according to the results in Fig. 5, the experimental hyperchaotic attractors phase trajectories of the DMHM with different parameters are displayed in Fig. 16. Then according to the results in Fig. 9, the experimentally displayed quasiperiodic, periodic and chaotic attractors of the DMHM are shown in Fig. 17. Similarly, corresponding to the results in Fig. 11, the experimental phase diagram showing a variety of complex coexistence phenomena are displayed in Fig. 18. The phase diagrams and time-series obtained from the experiments of the microcontroller-based DMHM system confirm all the predicted dynamic behaviors. Therefore, the digital experiments perfectly validate the numerical results, which manifest the feasibility of the hardware implementation for the presented locally active discrete memristor and the DMHM system. 


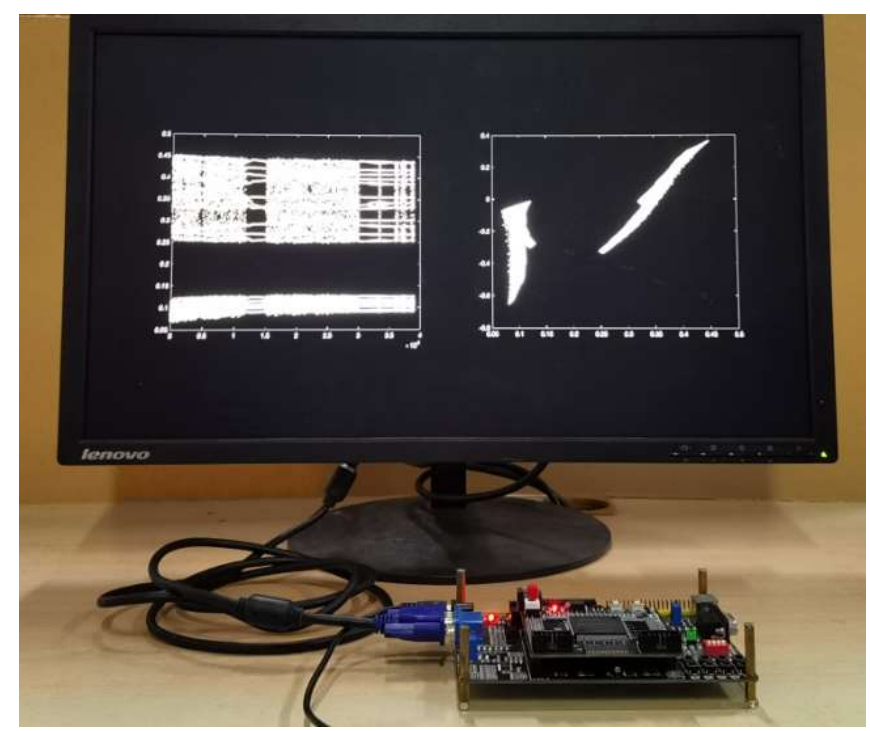

Fig. 15 The experimentally displayed transient chaotic voltage sequences and the attractor of the DMHM with parameters $b=0.5, r=0.6$ and $n=[1,40000]$

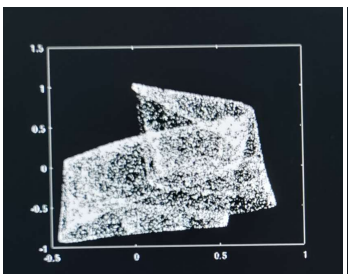

(a)

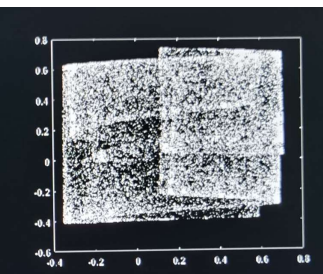

(b)

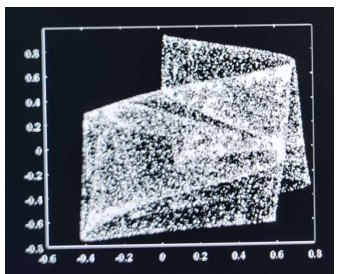

(c)

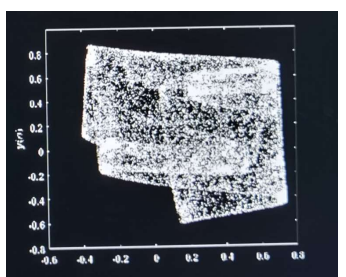

(d)

Fig. 16 The experimentally displayed hyperchaotic attractors of the DMHM with parameters $a=10, b=0.7$, $x(0)=0, y(0)=0.1, z(0)=2$, and $\mathbf{a} r=-0.8, \mathbf{b} r=-0.55$, $\mathbf{c} r=-0.1$ and $\mathbf{d} r=0.23$

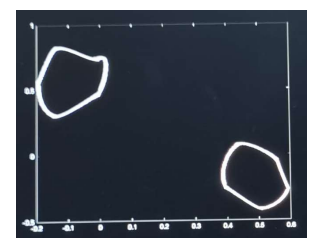

(a)

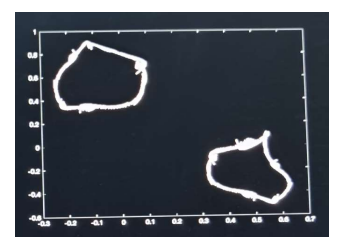

(b)

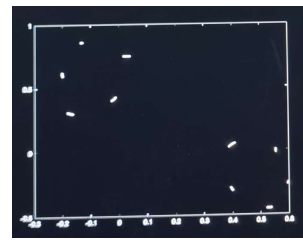

(c)

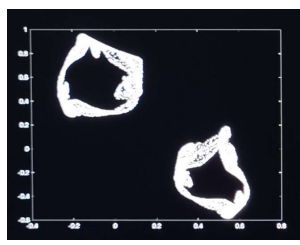

(d)

Fig. 17 The experimentally displayed quasi-periodic, periodic and chaotic attractors of the DMHM with parameters $b=0.5$ and $\mathbf{a} r=0.6, \mathbf{b} r=0.65, \mathbf{c} r=0.75, \mathbf{d} r=0.8$

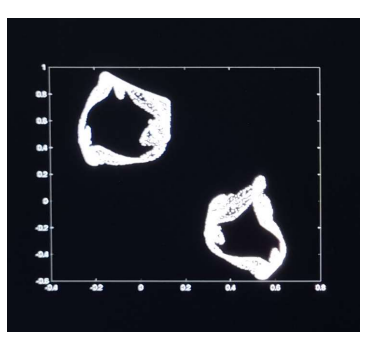

(a)

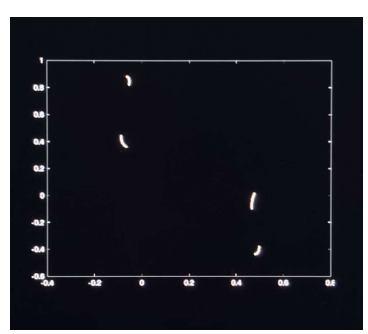

(b)

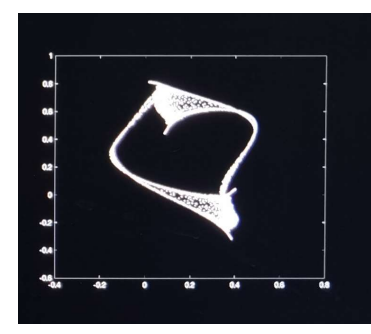

(c)

Fig. 18 The experimentally displayed coexisting attractors of the DMHM with parameters $r=0.8, \mathrm{~b}=0.5$ and initial values a $x(0)=0, y(0)=0.1, z(0)=2, \mathbf{b} x(0)=0, y(0)=0.1, z(0)=0, \mathbf{c} x(0)=0, y(0)=0.1, \mathrm{z}(0)=-2$ 


\section{Conclusion}

In this paper, a locally active discrete memristor model has been proposed for the first time and proved to satisfy the three fingerprints characteristics of generalized memristor. Then, the locally active discrete memristor is introduced into the two-dimensional generalized square map (2D-GSM), and the locally active discrete memristive hyperchaotic map (DMHM) is obtained. The DMHM shows different complex dynamics behaviors. Numerical simulations and hardware experiments have led to the following results:

(a) Compared with the original system, the DMHM expands its hyperchaotic region after adding the locally active discrete memristor, so it can produce more complex behaviors.

(b) The DMHM has quasi periodic state and periodic state before entering chaos, and the quasi periodic region is wider about 16 times than that of the original 2D-GSM.

(c) The dynamical behaviors of the DMHM system are affected by initial values, which leads to the appearance of coexisting attractors. Besides, the effective value range of parameter $r$ in the DMHM is much wider than that of the 2D-GSM.

(d) The DMHM has the phenomenon of transient chaos and transient period alternating in the time series, that is, the system presents different states due to different iteration times.

(e) The DMHM has antimonotonicity behavior with two bubble trees, and periodic orbits can be created and then annihilated via reverse period-doubling bifurcation scenarios as a bifurcation control parameter slowly changes.

Finally, the effectiveness of the DMHM system is verified by FPGA hardware. These results show that the locally active discrete memristor model is worthy for further study, and it can be applied to pseudo-random number generator or secure communication as well. Its application prospects in the field of traditional engineering are broader due to its many useful features. The next work should be the stability analysis and its application in discrete neural network.

Acknowledgements This work was supported by the National Key Research and Development Program of China under Grant No.2018AAA0103300 and the National Natural Science Foundations of China under Grants No. 62171401 and 62071411.

\section{Declarations}

Data Availability The datasets generated during and/or analyzed during the current study are available from the corresponding author on reasonable request.

Conflict of interest The authors declare that they have no conflicts of interest.

\section{References}

1. Chua, L.: Memristor-the missing circuit element. IEEE Transactions on Circuit Theory. 18(5), 507$519(1971)$

2. Strukov, D.B., Snider, G.S., Stewart, D.R.: The missing memristor found. Nature. 453(7191), 80-83 (2008) 
3. Valov, I., Linn, E., Tappertzhofen, S., Schmelzer, S.: Nanobatteries in redox-based resistive switches require extension of memristor theory. Nat Commun. 4, 1771 (2013)

4. Zhao, B., Xiao, M., Zhou, N.: Synaptic learning behavior of a TiO2 nanowire memristor. Nanotechnology. 30, 425202 (2019)

5. Zhang, Y., Zhuang, J., Xia, Y., Bai, Y., Cao, J., Gu, L.: Fixed-time synchronization of the impulsive memristor-based neural networks. Commun Nonlinear Sci Numer Simul. 77, 40-53 (2019)

6. Wang, C., Xiong, L., Sun, J., Yao, W.: Memristor-based neural networks with weight simultaneous perturbation training. Nonlinear Dyn. 95, 2893-2906 (2019)

7. Nagamani, G., Rajan, G., Zhu, Q.: Exponential state estimation for memristor based discrete-time BAM neural networks with additive delay components. IEEE Transactions on Cybernetics. 50(10), 4281-4292 (2020)

8. Duan, S., Hu, X., Dong, Z., Wang, L., Mazumder, P.: Memristor-based cellular nonlinear/neural network: design, analysis, and applications. IEEE Transactions on Neural Networks and Learning Systems. 26(6), 1202-1213 (2015)

9. Kim, H., Sah, M., Yang, C., Cho, S., Chua, L.: Memristor emulator for memristor circuit applications. IEEE Trans Circuits Syst I. 59(10), 2422-2431 (2012)

10. Rziga, F., Mbarek, K., Ghedira, S., Besbes, K.: An efficient Verilog-A memristor model implementation: simulation and application. J Comput Electron. 18, 1055-1064 (2019)

11. Wang, Y., Ma, J., Xu, Y., Wu, F., Zhou, P.: The electrical activity of neurons subject to electromagnetic induction and Gaussian white noise. Int J Bifurc Chaos. 27(2), 1750030 (2017)

12. Lai, Q., Wan, Z.Q., Kuate, P.D.K., Fotsin, H.: Coexisting attractors, circuit implementation and synchronization control of a new chaotic system evolved from the simplest memristor chaotic circuit. Communications in Nonlinear Science and Numerical Simulation. 89, 105341 (2020)

13. Wang, C.H., Lin, H.R., Sun, J.R., Zhou, L., Zhou, C., Deng, Q.L.: Research Progress on Chaos, Memory and Neural Network Circuits Based on Memristor. Journal of Electronics and Information Technology. 42(4), 795-810 (2020)

14. Li, H., Hua, Z., Bao, H., Zhu, L., Chen, M., Bao, B.: Two-Dimensional Memristive Hyperchaotic Maps and Application in Secure Communication. IEEE Transactions on Industrial Electronics. 68(10), 9931-9940, (2021)

15. Jin, P.P., Wang, G.Y., Iu, H.H.C.: A locally active memristor and its application in a chaotic circuit. IEEE Transactions on Circuits and Systems II: Express Briefs. 65(2), 246-250 (2018)

16. Chang, H., Wang, Z., Li, Y.X.: Dynamic analysis of a bistable bi-local active memristor and its associated oscillator system. International Journal of Bifurcation and Chaos. 28(8), 1850105 (2018)

17. Weiher, M., Herzig, M., Tetzlaff, R.: Pattern formation with locally active S-type NbOx memristors. IEEE Transactions on Circuits and Systems I: Regular Papers. 66(7), 2627-2638 (2019)

18. Lin, H.R., Wang, C.H., Sun, Y., Yao, W.: Firing multistability in a locally active memristive neuron. Nonlinear Dyn. 100, 3667-3683 (2020)

19. Li, Z.J., Zhou, H.Y., Wang, M.J., Ma, M.L.: Coexisting firing patterns and phase synchronization in locally active memristor coupled neurons with HR and FN models. Nonlinear Dyn. 104, 1455-1473 (2021)

20. Chew, Z.J., Li, L.: A discrete memristor made of $\mathrm{ZnO}$ nanowires synthesized on printed circuit board. Materials Letters. 91, 298-300 (2013) 
21. Karthikeyan, A., Rajagopal, K.: FPGA implementation of fractional-order discrete memristor chaotic system and its commensurate and incommensurate synchronisations. Pramana-Journal of Physics. 90(1), 14 (2018)

22. He, S.B., Sun, K.H., Peng, Y.X., Wang, L.Y.: Modeling of discrete fracmemristor and its application. AIP Advances. 10(1), 015332 (2020)

23. Peng Y. X., He, S.B., Sun, K.H.: Chaos in the discrete memristor-based system with fractional-order difference. Results in Physics. 24, 104106 (2021)

24. Peng, Y.X., He, S.B., Sun, K.H.: A higher dimensional chaotic map with discrete memristor. AEU International Journal of Electronics and Communications. 129, 153539 (2021)

25. Bao, B.C., Li, H.Z., Wu, H.G., Zhang, X., Chen, M.: Hyperchaos in a second-order discrete memristor-based map model. Electronics Letters. 56, 769-770 (2020)

26. Ma, J., Li, A.B., Pu, Z.S., Yang, L.J., Wang, Y.Z.: A time-varying hyperchaotic system and its realization in circuit. Nonlinear Dyn. 62, 535-541 (2010)

27. Peng, Y.X., Sun, K.H., He, S.B.: A discrete memristor-based chaotic map. Chaos Solitons \& Fractals. 137, 109873 (2020)

28. Adhikari, S.P., Sah, M., Kim, H., Chua, L.: Three fingerprints of memristor. IEEE Trans Circuits Syst I. 60(11), 3008-3021 (2013)

29. Chua, L.O.: If it's pinched it's a memristor. Semicond. Sci. Technol. 29(10), 104001 (2014)

30. He, S., Sun, K., Wang, H.: Complexity analysis and DSP implementation of the fractional-order lorenz hyperchaotic system. Entropy. 17, 8299-8311 (2015)

31. Dawson, S.P., Grebogi, C., Yorke, J.A., Kan, I., Koçak, H.: Antimonotonicity: inevitable reversals of period-doubling cascades. Physics Letters A .162(3), 249-254 (1992)

32. Parlitz, U., Lauterborn, W.: Superstructure in the bifurcation set of the Duffing equation. Phys. Lett. A. 107(8), 351-355 (1985)

33. Parlitz, U., Lauterborn, W.: Period-doubling cascades and devil's staircases of the driven van der Pol oscillator. Phys. Rev. A. 36, 1428 (1987)

34. Kocarev, L., Halle, K., Eckert, K., Chua, L.: Experimental observation of antimonotonicity in Chua's circuit. Int J Bifurc Chaos. 3(4), 1051-1055 (1993)

35. Kengne, J., Negou, A.N., Tchiotsop, D.: Antimonotonicity, chaos and multiple attractors in a novel autonomous memristor-based jerk circuit. Nonlinear Dyn. 88, 2589-2608 (2017)

36. Signing, V.R.F., Kengne, J., Pone, J.R.M.: Antimonotonicity, chaos, quasi-periodicity and coexistence of hidden attractors in a new simple 4-D chaotic system with hyperbolic cosine Nonlinearity. Chaos, Solitons and Fractals. 118, 187-198 (2019) 